\title{
A Pilot Clinical Study on Thiamine Hydrochloride as a New Mosquito Repellent: Determination of the Minimum Effective Dose on Human Skin
}

\author{
Alia Badawi, Mai El Halawany, and Randa Latif* \\ Department of Pharmaceutics \& Industrial Pharmacy, Faculty of Pharmacy, Cairo University; Kasr El Eini Street, \\ Cairo 11562, Egypt. \\ Received July 1, 2019; accepted November 8, 2019
}

\begin{abstract}
Thiamine hydrochloride has been suggested as a natural, safe yet effective alternative for chemical insect repellents. However, there is a demand for a reassessment of the minimum required dose that is sufficient to perform a topical repellency on the human skin. Therefore, the purpose of the current work is to establish a dose-response curve from which the effective dose (ED) is calculated. A series of increasing concentrations of thiamine hydrochloride were applied to the forearm of adult volunteers, the number of bites was counted and the percent repellency calculated accordingly. Data of percent repellency were converted to probit values which were plotted against log doses. A linear relation was obtained from the dose-response curve with an $r^{2}=0.958$. Statistical validation of the equation was tested through linear regression analysis, where the slope and intercept were found significant from zero. No significant difference was shown between observed and expected responses $(p>0.05)$. ED 50 and $99.9 \%$ were computed from the linear equation and found to be 4.57 and $344 \mathrm{mg}$, respectively. This finding can be supported by future works in which a proper formulation of thiamine hydrochloride in the respective doses would be presented. One can get prolonged safe protection against insect bites.
\end{abstract}

Key words thiamine hydrochloride; mosquitoes repellent; probit plane analysis; skin protection; preformulation

\section{INTRODUCTION}

Since ancient times, mosquitoes spread not only in tropical or subtropical regions but all over the planet. Nowadays, the phenomenon of global warming has encouraged more extensive growth of different species of mosquitoes. The actual risk lies in female insects which are nourished on blood meals taken from humans. In order to complete their meals, they inject saliva into the host, the latter may cause local discomfort, irritation and moreover transmission of contagious diseases. ${ }^{1)}$

The first line control of mosquitoes' bites has been executed through the use of insecticides. However, due to the known hazards of these chemical agents on human health, ${ }^{2,3)}$ another self-protection approach has emerged which depends on applying some topical pharmaceutical formulae on the exposed skin areas. These products have been named as insect repellents and have been considered as a major component of personal protection strategy. Up till now they provide remarkable measures to minimize the risk of mosquitoes borne diseases. ${ }^{4,5)}$ Basically, most repellents work on creating an odor barrier due to evolution of volatile substances. The latter have the potential of moving away mosquitoes from the host. ${ }^{6}$ )

The most worldwide used insect repellent was $N, N$-diethylmeta-toluamide (DEET) which was known for its strong repellent activity. ${ }^{7,8)}$ However, recent studies reported severe adverse reactions including encephalopathy in children, urticaria syndrome, anaphylaxis, hypotension and decreased heart rate. ${ }^{9)}$ It may also induce neuronal degeneration in brain ${ }^{10)}$ due to permeation through human skin. ${ }^{11)}$

Scientists were then directed towards using natural products as volatile and essential oils which are known for their repellent activity, Citronella oil, Eucalyptus oil and others were used. ${ }^{12)}$ However, these oils had short duration of action, and might cause allergic reactions upon oxidation of certain components. $^{13)}$

Thiamine hydrochloride (or vitamin $\mathrm{B}_{1}$ ) with molecular formula $\mathrm{C}_{12} \mathrm{H}_{18} \mathrm{Cl}_{2} \mathrm{~N}_{4} \mathrm{O}$ (IUPAC name: 2-[3-[(4-amino2-methylpyrimidin-5-yl)methyl]-4-methyl-1,3-thiazol-3ium-5-yl] ethanol; chloride; hydrochloride) (Fig. 1) is known to be essential in human carbohydrate metabolism.

Its deficiency in the body may lead to beri-beri syndrome. So its main medicinal use is in vitamin $\mathrm{B}_{1}$ deficiency. ${ }^{14)}$ Surprisingly, scientists noticed that long term treatment with oral thiamine hydrochloride was accompanied by some recorded insect repellent activity. Researchers explained that oral thiamine hydrochloride is normally excreted through pores of the skin. When taken in large dose, it appears on the skin along with perspiration in sufficient amount to be effective as mosquito repellent. ${ }^{15)}$ In a U.S. patent assigned to Roentsch et al. researchers were directed to use it topically at concentration range from 0.01 to $30 \%(\mathrm{w} / \mathrm{v}){ }^{16)}$ Spiegel LS and Spiegel MA in another U.S. patent explored the possibility of formulating topical thiamine along with other oils and sunscreen agents. ${ }^{17)}$

From this point of departure, we suggest the possibility of formulating a topical mosquito repellent containing thiamine<smiles>Cc1ncc(C[n+]2csc(CCO)c2C)c(N)n1</smiles>

Fig. 1. 2D Structure of Thiamine Hydrochloride 
hydrochloride. Normally, its dose is optimized according to the pharmacokinetic data recorded after oral or parenteral administration. Therefore, a demand for dose optimization is required to be established, especially if it has to perform a new pharmacological effect on the skin.

So the present work will be dedicated to the determination of the dose-activity relationship of thiamine hydrochloride as a non-specific mosquito repellent on human volunteers. The work will be executed through the estimation of the dose-response line and then calculating the repellent dose corresponding to the percent protection $50\left(\mathrm{ED}_{50}\right)$, and $99.9 \%$ $\left(\mathrm{ED}_{99.9}\right)$. The established dose will then be applied in a following work to formulate an extended release topical formulation.

The current work is done according to the WHO guidelines that were released in 2009. They provide specific and standardized procedures and criteria for efficacy testing and evaluation of mosquito repellents on human skin. ${ }^{18)}$

The protocol of the present work was approved by Experiments and Advanced Pharmaceutical Research Unit (EAPRU) as well as the ethical health committee (PT1446), Faculty of Pharmacy, Cairo University.

\section{MATERIALS AND METHODS}

Chemicals Thiamine hydrochloride (gift from Amoun Pharmaceutical Company, Obour city, Cairo, Egypt), Absolute Ethanol (analytical grade).

Preparation of the Test Solution Since thiamine hydrochloride (Fig. 1) is insoluble in absolute ethanol, serial dilutions of the drug were prepared in $90 \%$ ethanol. A volume of one $\mathrm{mL}$ was used in concentrations of 2,4 and $5 \mathrm{mg} / \mathrm{mL}$. A negative control of one $\mathrm{mL}(90 \%)$ ethanol was also used for comparison.

Study Subjects The study was conducted in the institute of medical entomology (El Dokki, Giza, Egypt). A total of 8 healthy subjects were selected for the study. Subjects' selection was based on the following inclusion criteria:

-Adults healthy subjects

-Subjects were asked not to use fragrance or tobacco $12 \mathrm{~h}$ prior to the experiment

-Subjects agreed to undergo the test on increasing concentrations of thiamine hydrochloride

Some subjects were excluded from the study depending on the following exclusion criteria:

-Atopic subjects with reported history of severe hypersensitivity reactions to food and drugs

-Subjects that did not agree to complete the test at all concentrations

Each participant was informed about the study and signed an informed consent.

Study Design All volunteers participating in the experiment $(n=8)$ were subjected through a simple randomization technique to increasing concentrations of thiamine hydrochloride solution and exposed to mosquitoes' bites. The test was performed three times on each subject and obtained results were mean of three determinations done at each drug concentration. Safety of the applied solution was assessed by monitoring subjects with respect to appearance of any signs and/or symptoms of adverse reaction on the skin (erythema, burning sensation, dryness, itching). The stability of the solution was determined from comparing results after three times repetition
Table 1. Applied Doses of Thiamine Hydrochloride and the Corresponding Cumulative Doses Spread on Tested Area

\begin{tabular}{ccc}
\hline \hline Experiment number & Applied dose (mg/) & Cumulative dose (mg) \\
\hline 1 & 0 (Control) & 0 \\
2 & 2 & 2 \\
3 & 4 & 6 \\
4 & 4 & 10 \\
5 & 5 & 15 \\
6 & 5 & 20 \\
\hline
\end{tabular}

of the test during successive days.

The forearm of the volunteer under test was marked with a marker pen on the specified area (about $600 \mathrm{~cm}^{2}$ ) between the wrist and elbow. The site of application was washed with water and unscented soap then $70 \%$ ethanol. A control consisting of $1 \mathrm{~mL}$ of $90 \%$ ethanol was applied by a pipette on the site of application then was left to dry for $1 \mathrm{~min}$. The forearm was inserted in the specified cage, the volunteer was asked not to move the hand under test to avoid disturbing mosquitoes. The number of mosquitoes that landed or bit in $30 \mathrm{~s}$ was counted. At least 10 or more mosquitoes had to land or bite in the specified time so that the test could count. The same steps were repeated for the next series of concentrations (w/v) of thiamine hydrochloride, one $\mathrm{mL}$ at each application (Table 1). Volunteers were asked not to rinse their hands throughout the experiment (after the application of each concentration) as doses were applied in a cumulative manner. Another $1 \mathrm{~mL}$ of $90 \%$ ethanol (control) was applied on the other hand (unused one during the experiment) at the end of the run to verify that the number of landings and/or bites was approximately $\geq 10$ per $30 \mathrm{~s}$, as was observed at the beginning of the experiment. Results were recorded for each volunteer separately.

Specifications for Mosquitoes Species chosen for the experiment was Culex pipines as this is a familiar species in Egypt. They were brought from the institute of medical entomology (El Dokki). Testing experiments and observations were performed at night as this was the period of highest mosquitoes' biting activity.

Standardized mosquito rearing and laboratory testing conditions were applied to ensure the reliability and reproducibility of data as follows: Mosquitoes were reared, maintained and tested at $27 \pm 2{ }^{\circ} \mathrm{C}, \geq 80 \pm 10 \%$ relative humidity, and a 12:12 (light:dark) photoperiod. They were put in a cage (size of $40 \times 40 \times 35 \mathrm{~cm}$ ) with a solid bottom and top, screen or netting on the back, a clear side view, and a fabric sleeve for access on the front (Fig. 2). Each cage contained 50 mosquitoes. Adult female mosquitoes were the candidates for the experiment. They were not subjected to blood meals $12 \mathrm{~h}$ prior to the experiment. Only sugar solution was allowed as feeding. Age of mosquitoes was between 5 to $15 \mathrm{~d}$ post emergence.

It has to be noted that a single test is said to be completed when one volunteer is subjected to the same mosquito batch after application of five successive concentrations of thiamine hydrochloride solution on the same day. The time for exposure to mosquitoes at each concentration was $30 \mathrm{~s}$. The same test was repeated (sequentially for three consecutive days) for each volunteer with the same sequence, three times using different batches of mosquitoes.

Statistical Analysis of Data Data were analyzed using probit-plane regression model, ${ }^{19-21)}$ which is a regression 

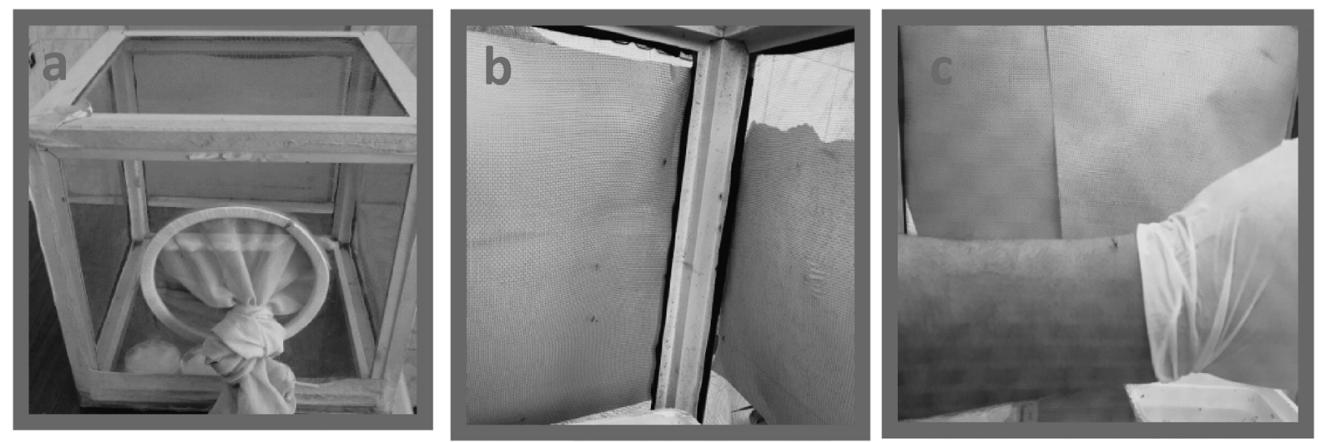

Fig. 2. Images for (a) Empty Cage, (b) Cage with Reared Female Mosquitoes, (c) Instant of the Insect Landing on the Forearm

Table 2. Test for Repellency of Thiamine Hydrochloride on Human Subjects

\begin{tabular}{|c|c|c|c|c|c|c|c|c|c|c|c|c|}
\hline \multirow{3}{*}{ Volunteer No. } & \multicolumn{12}{|c|}{ Number of bites and $\%$ repellency for each of the stated concentrations in $\mathrm{mg} / \mathrm{mL}$} \\
\hline & \multicolumn{2}{|c|}{0 (Control) } & \multicolumn{2}{|c|}{2} & \multicolumn{2}{|c|}{6} & \multicolumn{2}{|r|}{10} & \multicolumn{2}{|c|}{15} & \multicolumn{2}{|c|}{20} \\
\hline & $\begin{array}{l}\text { No of } \\
\text { bites }^{(a)}\end{array}$ & $\begin{array}{c}\% \\
\text { repellency }\end{array}$ & $\begin{array}{l}\text { No of } \\
\text { bites }^{a)}\end{array}$ & $\begin{array}{c}\% \\
\text { repellency }\end{array}$ & $\begin{array}{l}\text { No of } \\
\text { bites }^{a)}\end{array}$ & $\begin{array}{c}\% \\
\text { repellency }\end{array}$ & $\begin{array}{l}\text { No of } \\
\text { bites }^{a)}\end{array}$ & $\begin{array}{c}\% \\
\text { repellency }\end{array}$ & $\begin{array}{l}\text { No of } \\
\text { bites }^{a)}\end{array}$ & $\begin{array}{c}\% \\
\text { repellency }\end{array}$ & $\begin{array}{l}\text { No of } \\
\text { bites }^{a)}\end{array}$ & $\begin{array}{c}\% \\
\text { repellency }\end{array}$ \\
\hline 1 & $15 \pm 1.00$ & 0 & $10 \pm 1.00$ & 33.3 & $7 \pm 2.00$ & 53.3 & $4.7 \pm 0.57$ & 68.9 & $3 \pm 2.64$ & 80 & $2 \pm 1.73$ & 86.7 \\
\hline 2 & $12 \pm 1.89$ & 0 & $8 \pm 2.64$ & 33.3 & $6 \pm 1.73$ & 50 & $3 \pm 1.00$ & 75 & $2 \pm 1.00$ & 83.3 & $1.3 \pm 0.57$ & 88.9 \\
\hline 3 & $17 \pm 2.54$ & 0 & $14 \pm 2.64$ & 17.6 & $12 \pm 2.64$ & 29.4 & $8.3 \pm 5.03$ & 51 & $5 \pm 4.58$ & 70.6 & $4 \pm 4.35$ & 76.4 \\
\hline 4 & $12 \pm 1.67$ & 0 & $8 \pm 1.70$ & 33.3 & $4.3 \pm 0.57$ & 63.9 & $2.3 \pm 0.57$ & 80.6 & $2 \pm 0.00$ & 83.3 & $1 \pm 0.00$ & 91.7 \\
\hline 5 & $12 \pm 1.73$ & 0 & $6 \pm 0.00$ & 50.0 & $4.3 \pm 0.57$ & 63.9 & $4 \pm 1.00$ & 66.7 & $2 \pm 1.00$ & 83.3 & $1.3 \pm 0.57$ & 88.9 \\
\hline 6 & $16 \pm 4.47$ & 0 & $10 \pm 2.64$ & 37.5 & $8 \pm 2.00$ & 50 & $6 \pm 1.00$ & 62.5 & $3.3 \pm 0.57$ & 79.2 & $1.7 \pm 1.15$ & 89.6 \\
\hline 7 & $15 \pm 1.00$ & 0 & $11 \pm 1.00$ & 26.7 & $6 \pm 2.00$ & 60 & $3.5 \pm 0.70$ & 76.7 & $2 \pm 0.00$ & 86.7 & $1.5 \pm 0.70$ & 90 \\
\hline 8 & $20 \pm 1.82$ & 0 & $15 \pm 2.00$ & 25.0 & $11.5 \pm 0.70$ & 42.50 & $9 \pm 0.00$ & 55 & $4 \pm 2.00$ & 80 & $1 \pm 0.00$ & 95 \\
\hline Mean repellency & & 0 & & $32.1 \pm 3.09$ & & $51.6 \pm 4.13$ & & $67.0 \pm 3.69$ & & $80.8 \pm 1.67$ & & $88.4 \pm 1.91$ \\
\hline
\end{tabular}

a) Mean of three determinations \pm standard deviation (SD). b) Mean of 8 values at each concentration \pm standard error (S.E.). \% Repellency or protection at each dose $=$ (number of bites for the control-number of bites for the treated subject/number of bites for the control) $\times 100$. (a) Number of bites for the control is the mean of six determinations ( 3 recorded values at the beginning of the experiment +3 values at the end of the experiment).

method used to convert the classical sigmoid curve of the dose-response relationship into a linear relationship. This was done by transforming the values of the response (percent repellency) into probability units and doses were transformed to their logarithmic values, so the model would take the form:

$$
\boldsymbol{Y}=\boldsymbol{a}+\boldsymbol{b} \boldsymbol{X}
$$

Where $\boldsymbol{Y}$ represents percent repellency (transformed to probit values)

$\boldsymbol{X}$ represents $\log$ values of the selected doses

$\boldsymbol{a}$ is the intercept of the line obtained from graphical presentation of data

$\boldsymbol{b}$ is the slope of the line obtained from graphical presentation of data

Linear regression analysis was also performed to demonstrate the strength of correlation between percent repellency and $\log$ of the tested doses. Furthermore linear regression was adopted to assist results from probit-plane so as to prove the acceptability of the suggested linear model.

One way ANOVA test was applied to illustrate the probability of any significant difference to occur between the observed percent repellency and different tested doses of thiamine hydrochloride.

All data analyses were computed using IBM SPSS statistics 17.0 software.

\section{RESULTS AND DISCUSSION}

A total of 8 healthy adults subjects were recruited among family members, friends and colleagues. The age interval was 18-60. Equal number of male and female candidates was chosen. Thiamine hydrochloride solution (at all tested concentrations) proved to be completely safe as none of the participants suffered from any adverse reactions after the application of the test solution. No hypersensitivity reaction was recorded as well for all subjects. The three repeated determinations of the test at all concentrations gave insignificant difference in results $(p<0.05)$ which confirmed the stated stability of the test solution. $^{15)}$

Table 1 shows the number and amount of cumulative doses applied to each subject participating in the study. The recorded doses in the table were chosen because they gave responses between 10 and $90 \%$ repellency as per the WHO recommendations. ${ }^{18)}$ Doses which gave responses less than 10 or more than $90 \%$ were discarded.

Table 2 shows the percent repellency (protection) corresponding to each tested concentration $\mathrm{w} / \mathrm{v}$ of thiamine hydrochloride. As it is demonstrated, increasing its concentration was accompanied by a decrease in the number of bites and hence an increase in the percent protection or repellency of the drug against tested mosquitoes. Table 3 shows that the difference in percent repellency between the tested concentrations was significant $(p>0.05)$ except between 15 and 
Table 3. Calculated Least Significant Difference (LSD) of Percent Repellency at Different Tested Doses

\begin{tabular}{|c|c|c|c|c|c|c|}
\hline \multirow{2}{*}{ (I) Dose } & \multirow{2}{*}{ (J) Dose } & \multirow{2}{*}{ Mean difference (I-J) } & \multirow{2}{*}{ S.E. } & \multirow{2}{*}{ Sig. } & \multicolumn{2}{|c|}{ 95\% Confidence interval } \\
\hline & & & & & Lower bound & Upper bound \\
\hline \multirow[t]{4}{*}{2.00} & 6.00 & $-19.53375^{*}$ & 4.41882 & .000 & -28.5044 & -10.5631 \\
\hline & 10.00 & $-34.94250^{*}$ & 4.41882 & .000 & -43.9132 & -25.9718 \\
\hline & 15.00 & $-48.70250^{*}$ & 4.41882 & .000 & -57.6732 & -39.7318 \\
\hline & 20.00 & $-56.29625^{*}$ & 4.41882 & .000 & -65.2669 & -47.3256 \\
\hline \multirow[t]{4}{*}{6.00} & 2.00 & $19.53375^{*}$ & 4.41882 & .000 & 10.5631 & 28.5044 \\
\hline & 10.00 & $-15.40875^{*}$ & 4.41882 & .001 & -24.3794 & -6.4381 \\
\hline & 15.00 & $-29.16875^{*}$ & 4.41882 & .000 & -38.1394 & -20.1981 \\
\hline & 20.00 & $-36.76250^{*}$ & 4.41882 & .000 & -45.7332 & -27.7918 \\
\hline \multirow[t]{4}{*}{10.00} & 2.00 & $34.94250 *$ & 4.41882 & .000 & 25.9718 & 43.9132 \\
\hline & 6.00 & $15.40875^{*}$ & 4.41882 & .001 & 6.4381 & 24.3794 \\
\hline & 15.00 & $-13.76000^{*}$ & 4.41882 & .004 & -22.7307 & -4.7893 \\
\hline & 20.00 & $-21.35375^{*}$ & 4.41882 & .000 & -30.3244 & -12.3831 \\
\hline \multirow[t]{4}{*}{15.00} & 2.00 & $48.70250^{*}$ & 4.41882 & .000 & 39.7318 & 57.6732 \\
\hline & 6.00 & $29.16875^{*}$ & 4.41882 & .000 & 20.1981 & 38.1394 \\
\hline & 10.00 & $13.76000^{*}$ & 4.41882 & .004 & 4.7893 & 22.7307 \\
\hline & 20.00 & -7.59375 & 4.41882 & .095 & -16.5644 & 1.3769 \\
\hline \multirow[t]{4}{*}{20.00} & 2.00 & $56.29625^{*}$ & 4.41882 & .000 & 47.3256 & 65.2669 \\
\hline & 6.00 & $36.76250^{*}$ & 4.41882 & .000 & 27.7918 & 45.7332 \\
\hline & 10.00 & $21.35375^{*}$ & 4.41882 & .000 & 12.3831 & 30.3244 \\
\hline & 15.00 & 7.59375 & 4.41882 & .095 & -1.3769 & 16.5644 \\
\hline
\end{tabular}

Table 4. Parameter Estimates for the Linear Regression Analysis

\begin{tabular}{|c|c|c|c|c|c|c|c|}
\hline & \multirow{2}{*}{ Parameter } & \multirow{2}{*}{ Estimate } & \multirow{2}{*}{ S.E. } & \multirow{2}{*}{$Z$} & \multirow{2}{*}{ Sig. } & \multicolumn{2}{|c|}{$95 \%$ Confidence interval } \\
\hline & & & & & & Lower bound & Upper bound \\
\hline \multirow[t]{2}{*}{ PROBIT $^{a)}$} & Log dose & 1.64 & .176 & 9.102 & .000 & 1.255 & 1.945 \\
\hline & Intercept & $-1.07-$ & .166 & -6.374 & .000 & $-1.223-$ & $-.891-$ \\
\hline
\end{tabular}

a) PROBIT model: PROBIT $(\mathrm{p})=$ Intercept $+\mathrm{BX}($ Covariates $\mathrm{X}$ are transformed using the base 10000 logarithm.

Table 5. Comparison between Observed and Expected Percent Repellency

\begin{tabular}{ccccc}
\hline \hline Number & Log dose & Observed responses \% & Expected responses \% & Probability \\
\hline 1 & .301 & 32.09 & 28.24 & 0.28 \\
2 & .778 & 51.63 & 57.45 & 0.57 \\
3 & 1.000 & 67.04 & 70.64 & 0.70 \\
4 & 1.176 & 80.80 & 79.52 & 0.79 \\
5 & 1.301 & 88.39 & 84.72 & 0.84 \\
\hline
\end{tabular}

$20 \mathrm{mg} / \mathrm{mL}$ where the difference was not significant $(p<0.05)$. This gave indication of the localization of the extreme values which were $\geq 15 \mathrm{mg} / \mathrm{mL}$. Therefore concentrations up to $15 \mathrm{mg} / \mathrm{mL}$ could significantly exert a repellent effect.

According to results shown in Table 2, a relation between $\log$ of the tested doses and probit values corresponding to percent repellencies was drawn. ${ }^{22}$ As shown in Fig. 3, a linear relationship was established with an $r^{2}=0.958$. The equation of the obtained straight line was computed as $y=1.64 x-1.07$ with parameter estimates shown in Table 4, where the slope and the intercept are significantly different from zero $(p<0.05)$. This means that the percent repellency ( $Y$ variable $)$ and log the dose ( $x$ variable) have a significant correlation and that the straight line model equation is accepted. A further statistical analysis on the observed percent repellencies (observed responses) was done, where the latter were compared

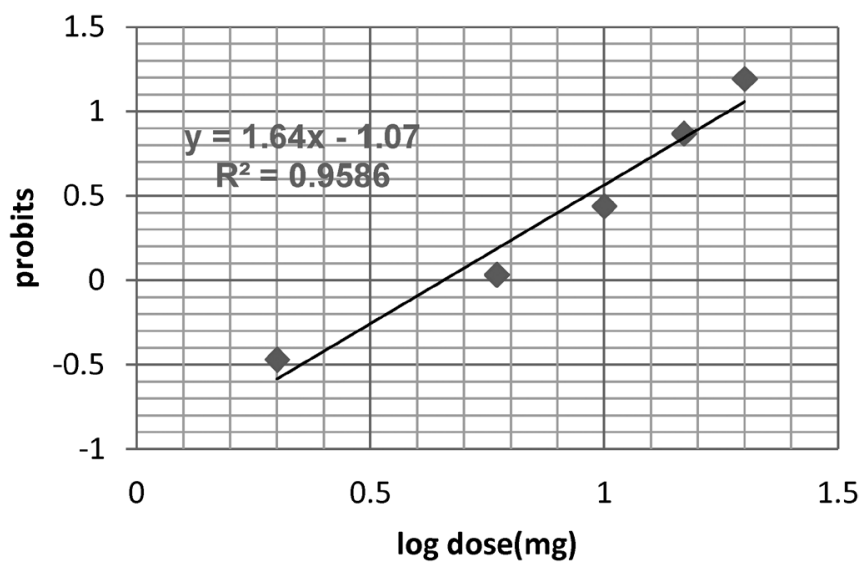

Fig. 3. Relation between Probit Transformed Responses and Logarithm of Doses 
to the expected responses to occur in order to fit the suggested experimental model. Table 5 shows that the difference between the observed and expected responses was non-significant at all tested concentrations of thiamine hydrochloride $(p>0.05)$. Such results confirm the validity of the established linear equation that fits the model. It has to be noted that the source of slight variation from the expected responses might arise from factors related to differences in subjects' skin $\mathrm{pH}$ and/or secretions. Other variations were probably got from different tendencies of insects to the same stimulus.

The established linear equation $(y=1.64 x-1.07)$ was used to estimate the $\mathrm{ED}_{50}$ and $\mathrm{ED}_{99.9}$ (effective dose that give 50 and $99.9 \%$ repellency against tested mosquitoes) by substituting for the $y$ vector by the probit values equivalent to 50 and $99.9 \%$ repellency and then calculating the values of the corresponding $x$ vector (representing the log of the dose). The calculated $\mathrm{ED}_{50}$ and $\mathrm{ED}_{99.9}$ were 4.57 and $344.02 \mathrm{mg}$, respectively. Lower and upper bound level were estimated as $3.62-5.5 \mathrm{mg}$ and $275.12-412.82 \mathrm{mg}$ for $\mathrm{ED}_{50}$ and $\mathrm{ED}_{99.9}$, respectively, at $95 \%$ confidence level.

\section{CONCLUSION}

Thiamine hydrochloride was shown to exert a repellent activity against Culex pipines. Through probit plane analysis model, the relation between the percent repellency and log doses was transformed to an equation of straight line. The validity of the experimental linear model was set through linear regression, where coefficients of the slope and intercept were found significant from zero. Furthermore no significant difference was stated between observed and expected percent repellencies. By simple substitution in the regression equation, a dose of 4.57 and $344 \mathrm{mg}$ of thiamine hydrochloride proved to exert 50 and $99.9 \%$ repellency, respectively. On the basis of this finding, future work can be dedicated to the production of a suitable controlled release topical formulation on the skin that can provide a prolonged outdoor safe protection against insect bites.

Acknowledgments The authors would like to thank members of the institute of medical entomology (El Dokki, Giza, Egypt) for their help and support to provide reared mosquitoes generation.

Conflict of Interest The authors declare no conflict of interest.

\section{REFERENCES}

1) Tolle MA. Mosquito-borne diseases. Curr. Probl. Pediatr. Adolesc.
Health Care, 39, 97-140 (2009).

2) Damalas CA, Eleftherohorinos IG. Pesticide exposure, safety issues, and risk assessment indicators. Int. J. Environ. Res. Public Health, 8, 1402-1419 (2011).

3) Sarwar M. Indoor risks of pesticide uses are significantly linked to hazards of the family members. Cogent. Med., 3, 1-10 (2016).

4) Bissinger BW, Roe RM. Tick repellents: past, present and future. Pesticide Biochem. Physiol., 96, 63-79 (2010).

5) Peterson C, Coats J. Insect repellents-past, present and future. Pesticide Outlook, 12, 154-158 (2001).

6) Islam J, Zaman K, Duarah S, Raju PS, Chattopadhyay P. Mosquito repellents: an insight into the chronological perspective and novel discoveries. Acta Trop., 167, 216-230 (2017).

7) Sudakin DL, Osimitz T. DEET. Hayes' hand-book of pesticide toxicology, third ed. (Krieger R ed.) Academic Press, New York, pp. 2111-2125 (2010).

8) Leal WS. The enigmatic reception of DEET - the gold standard of insect repellents. Curr. Opin. Insect Sci., 6, 93-98 (2014).

9) Antwi FB, Shama LM, Peterson RKD. Risk assessments for the insect repellents DEET and picaridin. Regul. Toxicol. Pharmacol., 51, 31-36 (2008).

10) Abdel-Rahman A, Dechkovskaia AM, Goldstein LB, Bullman SH, Khan W, El-Masry EM, Abou-Donia MB. Neurological deficits induced by malathion, DEET, and permethrin, alone or in combination in adult rats. J. Toxicol. Environ. Health A, 67, 331-356 (2004).

11) Wang T, Miller D, Burczynski F, Gu X. Evaluation of percutaneous permeation of repellent DEET and sunscreen oxybenzone from emulsion-based formulations in artificial membrane and human skin. Acta. Pharm. Sin. B, 4, 43-51 (2014).

12) Maia MF, Moore SJ. Plant-based insect repellents: a review of their efficacy, development and testing. Malar. J., 10 (Suppl. 1), S11 (2011).

13) Hagvall L, Karlberg AT, Christensson JB. Contact allergy to air-exposed geraniol: clinical observations and report of 14 cases. Contact Dermatitis, 67, 20-27 (2012).

14) Brayfield A. Martindale. The complete drug reference, thirtysixth ed., Pharmaceutical Press, United Kingdom, pp. 1940-1941, 1976-1977 (2009).

15) Marks MB. Stinging insects: allergy implications. Pediatr. Clin. North Am., 16, 177-191 (1969).

16) Roentsch E, Buderer M, Ford P. U.S. Patent Application Publication 20050181001A1 (2008) [Topical formulation for insect repellents].

17) Spiegel LS, Spiegel MA. U.S. Patent Application Publication 20070092546A1 (2007) [Thiamine based insect repellent].

18) Dept. of Control of Neglected Tropical Diseases, World Health Organization. Guidelines for efficacy testing of mosquito repellents for human skin., World Health Organization, Geneva (2009).

19) Finney DJ. Probit analysis, second ed. Cambridge University Press, Cambridge, England (1952).

20) Bliss CI. Obituary notices. Int. Stat. Rev., 48, 135-136 (1980).

21) Norton EC, Wang H, Ai C. Computing interaction effects and standard errors in logit and probit models. Stata J., 4, 154-167 (2004).

22) Rutledge LC, Wirtz RA, Buescher MD, Mehr ZA. Mathematical models of the effectiveness and persistence of mosquito repellent. $J$. Am. Mosq. Control Assoc., 1, 56-62 (1985). 